\title{
Evaluating two techniques for potentially increasing radial artery size in a follow-up study involving patients with peripheral vascular disease
}

\author{
Rana Hamidizadeh, MD (D) - Marcus Tholin, MD • Himat Vaghadia, MD • \\ Andrew Sawka, MD • Neil Ramsay, MD • Raymond Tang, MD • \\ Steven Lee, MD
}

Received: 13 July 2018/Revised: 22 July 2018/Accepted: 23 July 2018/Published online: 3 August 2018

(C) Canadian Anesthesiologists' Society 2018

\section{To the Editor,}

Radial arterial cannulation is an established procedure for continuous blood pressure monitoring. ${ }^{1}$ Ultrasound imaging can facilitate radial artery cannulation and is useful in situations such as hypotension when arterial diameter may be reduced because of vasoconstriction., ${ }^{2,3}$ Recently, Maheshwari et al. proposed two novel techniques to increase radial artery size by compressing the radial or ulnar artery distal to the cannulation site. ${ }^{3}$ Shao et al. investigated these techniques in 35 healthy volunteers, showing that compression of the distal radial artery, but not the ipsilateral ulnar artery, significantly increased the diameter of the radial artery. ${ }^{4}$ It is not known whether patients with peripheral vascular disease (PVD), where radial artery cannulation can be particularly difficult, would demonstrate a similar response. We therefore repeated this study in such patients to assess the potential for these techniques to improve success rates of ultrasoundguided radial artery cannulation.

This observational study was conducted at Vancouver General Hospital and was approved by the Clinical Research Ethics Board of the University of British Columbia (November 1, 2017: \#H17-02013). Informed written consent was obtained from 43 American Society of Anesthesiologists physical status II-IV patients with PVD, which was defined as having had a previous peripheral vascular procedure or currently presenting for one. Exclusion criteria included patients with previous surgery

R. Hamidizadeh, MD $(\bowtie) \cdot$ M. Tholin, MD · H. Vaghadia, MD . A. Sawka, MD · N. Ramsay, MD - R. Tang, MD - S. Lee, MD Department of Anesthesiology, Pharmacology and Therapeutics, University of British Columbia, 899 W 12th Ave, Vancouver, BC V5Z 1M9, Canada

e-mail: rana.hamidi@alumni.ubc.ca to the radial or ulnar artery, a history of an arteriovenous (AV) fistula, absent or anomalous arterial anatomy confirmed by ultrasound, a positive Allen's test, allergy to ultrasound gel, vascular procedures not due to PVD, and those presenting for emergency surgery.

An L25n/13-6 MHz linear transducer (SonoSite NanoMaxx Ultrasound System REF P12092-70/SN 04608K, FUJIFILM SonoSite Inc, Bothell, WA, USA) was used to visualize the radial artery $2-3 \mathrm{~cm}$ proximal to the radial styloid process. Radial artery measurements were taken at this location under the same three study conditions as used in a similar study by Shao et al.: i) control: no compression; ii) compression of the radial artery distally; and iii) compression of the ipsilateral ulnar artery 1-2 cm proximal to the distal wrist crease. Mechanical compression was performed/confirmed using an identical transducer. The order of these methods was randomized and an investigator who was blinded to the method used measured and recorded the vertical and horizontal diameter of the radial artery. The mean of these diameters was used in the statistical analysis. Measurements were taken on both wrists as long as the inclusion criteria continued to be fulfilled.

Over a two-month period, 62 patients were reviewed for potential eligibility. Of these, 19 were excluded (as described in the Figure) resulting in 43 patients (22 male, 21 female) being recruited. The mean (standard deviation [SD]) weight, systolic blood pressure, and diastolic blood pressure was $77.8(15.2) \mathrm{kg}, 133$ (21) $\mathrm{mmHg}$, and 68 (12) mmHg respectively. In 11 patients, only one wrist could be scanned (for reasons outlined in the Figure), giving us a radial artery sample size of 75 .

The mean (SD) uncompressed radial artery diameter was $2.3(0.7) \mathrm{mm}$. Neither distal radial artery compression nor ipsilateral ulnar artery compression increased the radial 


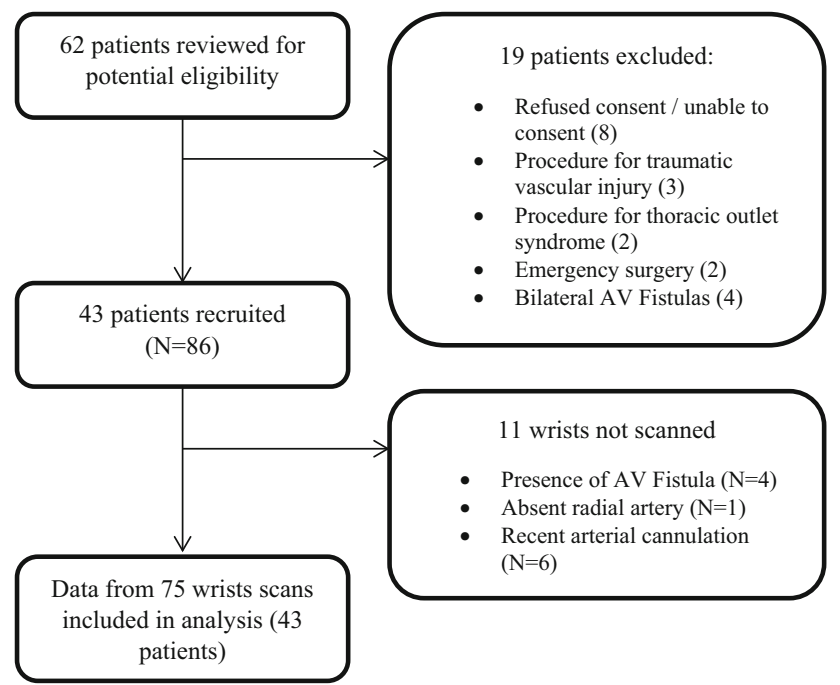

Figure Study flow chart

artery diameter. The mean difference with distal radial artery compression was $-0.02 \mathrm{~mm}$ (95\% confidence interval $[\mathrm{CI}],-0.02$ to $0.07 ; P=0.33$ ), while the mean difference with ulnar artery compression was $-0.04 \mathrm{~mm}$ (95\% CI, -0.01 to $0.09 ; P=0.95$ ).

In conclusion, we observed, in contrast to the findings of Shao et al., that neither compression of the distal radial artery nor the ulnar artery increased the size of the radial artery in patients with PVD. This may reflect the inability of diseased arteries in this patient population to respond to back pressure from a distally occluded vessel. It does not appear that these maneuvers will increase successful radial artery cannulation in patients with PVD.

Acknowledgements The authors thank Boris Kuzeljevic for his statistical consultation and analysis contributions.

Conflicts of interest None declared.

Editorial responsibility This submission was handled by Dr. Hilary P. Grocott, Editor-in-Chief, Canadian Journal of Anesthesia.

Funding This work was supported by Department of Anesthesiology funding only.

\section{References}

1. Zhou Q, Yao J, Chen Y, Lu J, Zhang X, Jiang W. Distal tourniquetfacilitated radial artery cannulation in adults - a double-blinded, prospective, randomized and controlled study. J Vasc Access 2015; 16: 158-62.

2. Gu WJ, Wu XD, Wang F, Ma ZL, Gu XP. Ultrasound guidance facilitates radial artery catheterization: a meta-analysis with trial sequential analysis of randomized controlled trials. Chest 2016; 149: 166-79.

3. Maheshwari P, Kelsheimer B, Maheshwari P. Novel techniques for increasing the size of the radial artery during ultrasound-guided cannulation. Can J Anesth 2016; 63: 499-500.

4. Shao E, Vaghadia H, Ramsay N, Tang R, Sawka A. Evaluation of two novel techniques for increasing radial artery size: a pilot study utilizing volunteers. Can J Anesth 2017; 64: 786-7. 